\title{
An Estimation Method of Representative Humanoids for Digital Human Simulation
}

\author{
Kihyo Jung \\ School of Industrial Engineering, University of Ulsan, Ulsan, 680-749
}

\begin{abstract}
Objective: The present study developed an estimation method of boundary zone representative humanoids(hereafter, $E B Z$ method) using descriptive statistics on the design target population. Background: The boundary zone method(hereafter, $B Z$ method) generates representative humanoids at a boundary zone that statistically accommodates a designated percent of the design target population; however, the BZ method has a practical limitation because it requires a large scale anthropometric database on the design target population. Method: The EBZ method developed in the present study consisted of 3 steps. In the first step, the boundary zone of accommodating a designated percent(e.g., 90\%) is formed under the assumption of normal distributions for anthropometric sizes. In the second step, cases that fall within the boundary zone are estimated using descriptive statistics(mean, standard deviation, and covariance) on the design target population. In the last step, K-mean cluster analysis is conducted for the cases, and representative humanoids are selected from each of clusters. Results: Evaluation results showed that mean accommodation percent of the EBZ method was 90.9\%(range: 90.8 91.1\%) which is similar to the target percent $(90 \%)$. In addition, standard deviation of accommodation percent for 100 repetitions was $0.1 \%$. Lastly, the number of representative humanoids generated by the $\mathrm{EBZ} \operatorname{method}(n=20)$ was similar to the $\mathrm{BZ} \operatorname{method}(n=$ 16). Conclusion: The EBZ method can generate representative humanoids which accommodate a designated percent of the design target population using descriptive statistics. Application: The EBZ method can be utilized in the generation of humanoids for ergonomic design and evaluation of products when the large scale anthropometric database on the design target population is not existed.
\end{abstract}

Keywords: Representative humanoid, Digital human simulation, Estimated boundary zone method, Multivariate accommodation

\section{Introduction}

가상환경상 인간공학적 평가 기술(digital human simulation; 이하, $D H S$ ) 은 제품의 사용성을 향상시키고 설계 과 정의 효율성을 제고시킬 수 있다. DHS는 대표인체모델과 설 계 대상 제품 간의 상호작용을 컴퓨터 상에서 평가하여 설계 상의 문제를 조기에 발견 및 보완할 수 있게 한다(Ryu et al., 2004; Nelson, 2001; Jimmerson, 2001; Thompson, 2001). 그로 인해, DHS는 순환적 설계 과정을 단축시킴으 로써 신속하고 경제적으로 사용자 친화적인 제품을 개발할 수 있게 한다(Chaffin, 2001).

가상환경상 평가를 위한 대표인체모델은 백분위 기법 (percentile method)을 이용해 생성되고 있다. 백분위 기법 은 백분위(예: $95^{\text {th }}$ percentiles)로 대표인체모델의 크기를 결정하기 때문에 대표인체모델 생성 절차가 간단하고 생성

Corresponding Author: Kihyo Jung. School of Industrial Engineering, University of Ulsan, Ulsan, 680-749.

Mobile: +82-10-5526-6381, E-mail: kjung@ulsan.ac.kr

Copyright@2013 by Ergonomics Society of Korea(pISSN:1229-1684 eISSN:2093-8462). All right reserved.

(c) This is an open-access article distributed under the terms of the Creative Commons Attribution Non-Commercial License(http://creativecommons.org/licenses/by-nc/3.0/), which permits unrestricted non-commercial use, distribution, and reproduction in any medium, provided the original work is properly cited. http://www.esk.or.kr 


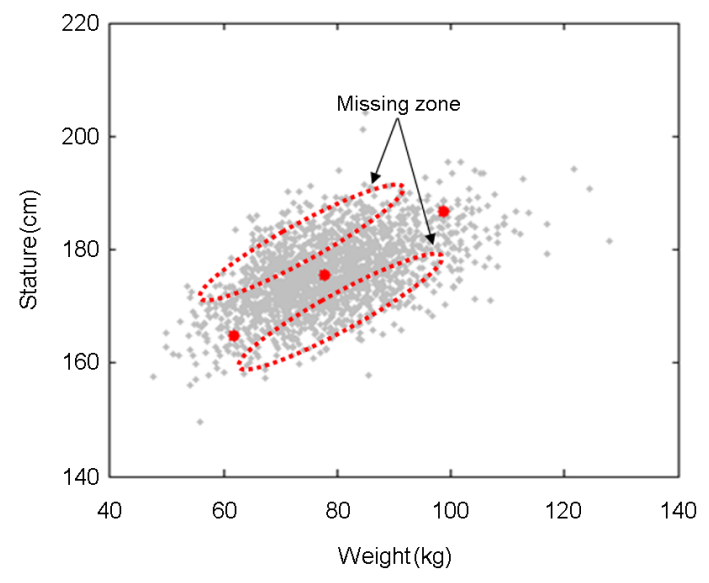

(a) Percentile representative humanoids

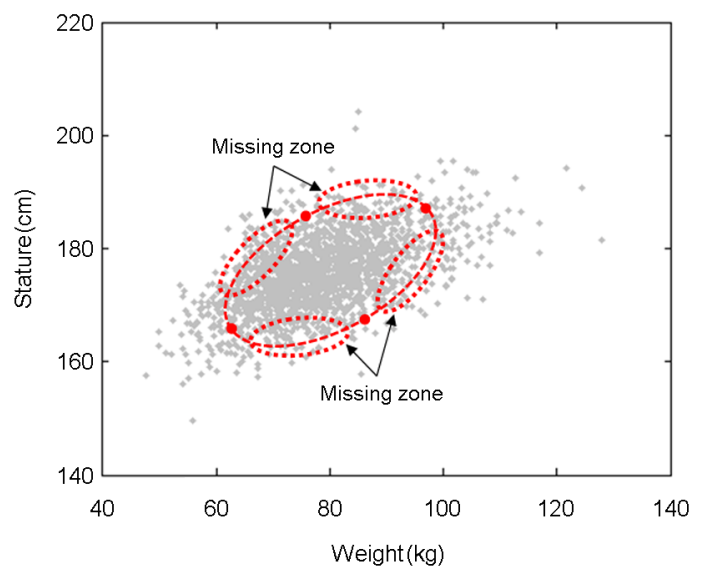

(b) Boundary representative humanoids

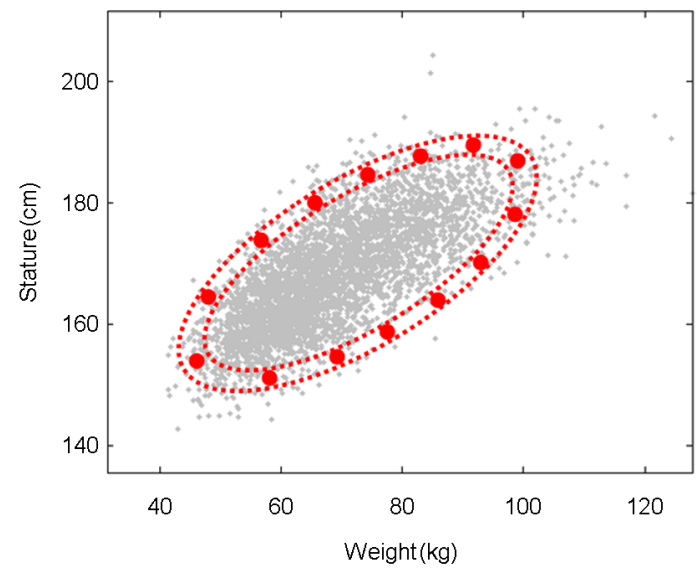

(c) Boundary zone representative humanoids

Figure 1. Comparison of representative humanoid generation methods(large dot: representative humanoids, small dot: design target users, source of raw data: Gordon et al.(1988))
개수(예: 3명)가 적은 장점이 있다(HFES 300, 2004). 그러 나 백분위 기법은 Figure 1.a에 나타낸 것과 같이 사용자의 다양한 인체크기를 대표하지 못하는 한계점이 있다(Roebuck et al., 1975; Porter et al., 2004).

백분위 기법의 한계점을 보완하기 위해 목표 비율(예: $90 \%)$ 의 설계 대상 사용자를 포함하는 경계선에서 대표인체 모델을 생성하는 기법(boundary method; 이하, Boundary 기법)이 개발되고 있다(Bittner et al., 1987; Meindl et al., 1993; Kim and Whang, 1997; Bittner, 2000). Boundary 기법은 Figure 2에 나타낸 것과 같은 3 단계 절차를 통해 대 표인체모델을 생성한다. 첫째 단계는 효율적으로 대표인체모 델을 생성하기 위해 자료 축약 기법(예: factor analysis)을 이용하여 인체변수를 소수(예: 2 3개) 의 공통요인으로 축약 한다. 둘째 단계는 선정된 공통요인 공간에서 목표 비율의 사용자를 포함하는 사각형의 경계선을 형성하고, 경계선의 모서리에 위치한 사용자를 대표인체모델로 결정한다. 마지막 단계는 공통요인 공간에서 정의된 대표인체모델의 크기를 공통요인과 인체변수 간의 통계적 연관관계를 나타내는 요 인적재량을 이용하여 인체크기로 변환한다.

Boundary 기법은 2 가지 측면의 한계점이 있다. 첫째, Boundary 기법은 효율적인 대표인체모델 생성을 위해 자료 축약 기법을 이용하여 설계 대상 사용자의 인체크기 분포를 대부분 설명 (예: variance explained $=80 \%$ )하는 소수의 공통요인을 추출한다. 그러나 자료 축약 과정에서 인체크기 의 손실(예: $20 \%$ )이 발생하여 사용자의 인체크기 다양성을 대표인체모델 생성에 반영하지 못하는 한계점이 있다(Jung et al., 2009a; Meunier, 1998). 둘째, Boundary 기법은 Figure 1.b에 나타낸 것과 같이 대표인체모델이 부재된 경계 선 상의 영역이 존재한다. 그로 인해, 설계 대상 사용자의 다 양한 인체크기 특성을 대표하지 못하는 한계점이 있다(Jung et al., 2009a).

최근 들어 Boundary 기법의 한계점을 보완하기 위해 경 계 영역에서 대표인체모델을 생성하는 기법(boundary zone method; 이하 $B Z$ 기법)이 개발되었으나 방대한 인체측정 원시자료가 필요한 한계점이 있다(Jung et al., 2009a). BZ 기법은 Figure 1.c와 같이 목표한 비율을 포함하는 경계 영 역을 통계적 이론에 근간하여 설정한 후 경계 영역 내부에 포함된 사용자 중에서 대표인체모델을 선발한다. $\mathrm{BZ}$ 기법은 목표한 비율을 이상적으로 충족하고 경계 영역 전반에 균형 분포된 대표인체모델을 생성함으로써 다양한 사용자의 인체 크기를 대표할 수 있다. 그러나 경계 영역 내부의 사용자 중 에서 대표인체모델을 선발해야 하기 때문에 경계 영역 내부 에 충분한 사용자가 포함될 수 있도록 인체측정자료가 방대 (예: $n \geq 3,000$ )해야 하는 한계점이 있다.

본 연구는 기술통계자료를 이용하여 설계 대상 사용자의 
Step 1: Extract factors by factor analysis

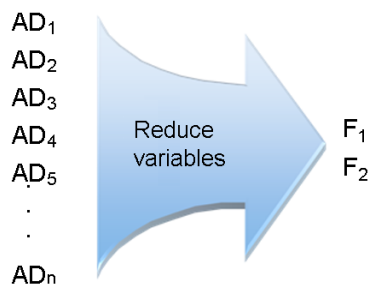

Step 2: Determine factor scores of representative humanoids (RHs)

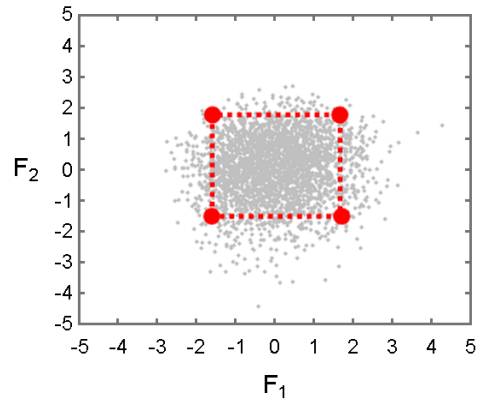

Step 3: Convert the factor scores to body sizes of RHs

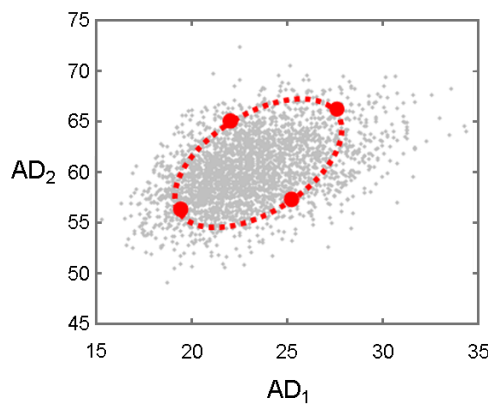

Figure 2. Generation process of boundary representative humanoids using Factor Analysis(AD: anthropometric dimension, F: factor, n: number of anthropometric dimensions; small dot: design target population, large dot: representative humanoids)

Step 1: Formation of a boundary zone

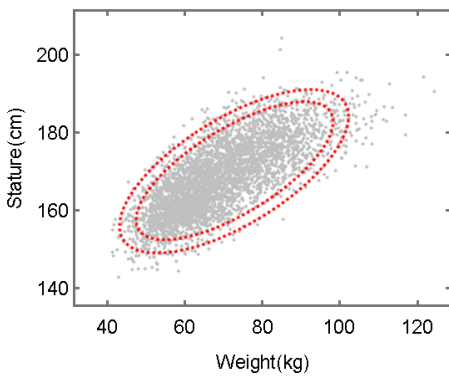

Step 2: Estimation of cases within the boundary zone

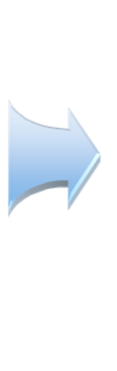

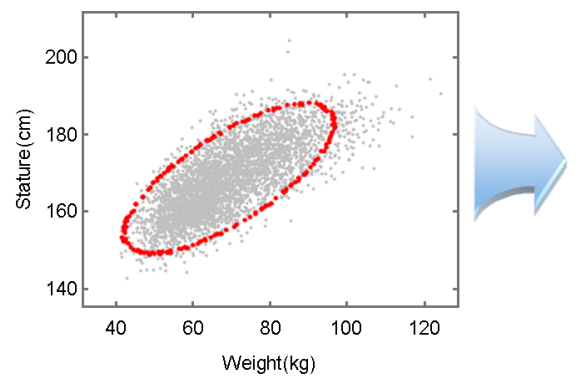

Step 3: Selection of RHs by cluster analysis

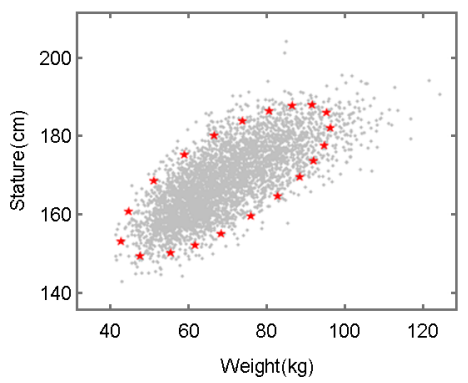

Figure 3. Generation process of estimated boundary zone representative humanoids(dotted line: boundary line, small dot: design target users, large dot: estimated users, star: representative humanoids)

다양한 인체크기를 대표하는 경계 영역 인체모델을 추정하 는 기법 (estimated boundary zone method; 이하, $E B Z$ 기 법)을 개발하였다. EBZ 기법은 설계 대상 사용자의 인체크 기에 대한 평균, 표준편차, 그리고 공분산을 이용하여 목표 한 비율을 이론적으로 충족하는 대표인체모델을 생성한다. 본 연구는 3 가지 척도(수용률, 이상치 비율, 그리고 대표인 체모델 개수)를 이용하여 $\mathrm{EBZ}$ 기법의 성능을 정량적으로 평가하였다.

\section{Estimation Method of Boundary Zone Representative Humanoids}

본 연구는 Figure 3 에 나타낸 것과 같은 3 단계 절차로 구 성된 $\mathrm{EBZ}$ 기법을 개발하였다. 첫째 단계는 인체크기에 대한 정규분포 가정에 근간하여 목표 비율을 수용하는 경계 영역
을 형성한다. 둘째 단계는 기술통계자료를 이용하여 경계 영 역 내부에 포함되는 사용자를 무작위로 추정한다. 마지막 단 계는 경계 영역 내부에 포함된 사용자에 대한 군집분석을 통해 군집의 중앙에 위치한 사용자들을 대표인체모델로 선 발한다.

\subsection{Formation of a Boundary Zone}

경계 영역은 목표 비율에 허용 비율을 가감하여 결정되는 2 개의 경계선으로 형성된다. 예를 들면, $90 \%$ 경계 영역은 목표 비율 $(90 \%)$ 에 허용 비율 $(1 \%)$ 을 가감하여 결정된 $89 \%$ 와 $91 \%$ 경계선으로 설정된다. 목표 비율의 사용자를 포함 하는 경계선은 정규분포를 따르는 인체크기의 표준화된 제 곱 거리(식 1)가 Chi-square 분포를 따른다는 기존 연구 (Jung et al., 2009a; Jung et al., 2009b; Johnson and Wichern, 1988)를 적용하여 파악된다. 예를 들면, 인체변수 가 2 개인 경우 사용자의 $89 \%$ 와 $91 \%$ 를 포함하는 경계선은 
식 1 에 의해 $\chi_{2}^{2}(1-0.89)=4.41$ 과 $\chi_{2}^{2}(1-0.91)=4.82$ 으 로 설정된다.

$$
D=(A D-\mu)^{T} \Sigma^{-1}(A D-\mu) \leq \chi_{n}^{2}(1-p)
$$

$$
\begin{array}{ll}
\text { where: } D & =\text { normalized squared distance } \\
A D & =n \text {-by- } 1 \text { body size matrix } \\
n & =\text { number of anthropometric dimensions } \\
\mu & =n \text {-by-1 average body size matrix } \\
\Sigma & =n \text {-by- } n \text { covariance matrix } \\
\chi_{n}^{2}(1-p) & =\text { Chi-squared value for } n \text { degree } \\
& \\
p & =\text { of freedom and } p \text { probability } \\
& \text { target accommodation percent }
\end{array}
$$

\subsection{Estimation of cases within the Boundary Zone}

경계 영역 내부에 포함된 사용자의 추정은 3 단계 절차를 통해 이루어진다. 첫째 단계는 설계 대상 사용자에 대한 기 술통계자료(평균, 표준편차)를 이용하여 사용자의 인체크기 를 무작위로 추정한다. 둘째 단계는 추정된 사용자가 경계 영역 내부에 포함되는지 여부를 기술통계자료(평균, 표준편 차, 공분산) 와 추정된 사용자의 인체크기를 식 1에 대입하여 판단한다. 예를 들면, 설계 대상 집단의 평균 신장 $169 \mathrm{~cm}$ (표준편차: 9.0), 평균 체중 $69 \mathrm{~kg}$ (표준편차: 13), 그리고 신 장-체중 공분산 85 일 때 신장과 체중이 $170 \mathrm{~cm}$ 와 $61 \mathrm{~kg}$ 인 사용자는 표준화된 제곱거리가 2.40 로서 경계 영역의 범위 (4.41 4.82)를 벗어난다. 반면, 신장과 체중이 $180 \mathrm{~cm}$ 와 $63 \mathrm{~kg}$ 인 사용자는 표준화된 제곱 거리가 4.58 로서 경계 영 역 내부에 포함된다. 마지막 단계는 경계 영역 내부에 포함 된 사용자를 지정된 수(예: 1,000명)에 도달할 때까지 반복 적으로 생성한다.

\subsection{Selection of representative humanoids by cluster analysis}

경계 영역 내부에 포함된 사용자들은 Figure 3에 나타낸 것과 같이 유사한 인체크기를 가질 수 있다. 따라서 경계 영 역 내부의 사용자에 대한 군집분석을 통해 대표인체모델 생 성 개수를 최적화하는 것이 필요하다. $\mathrm{EBZ}$ 기법은 $\mathrm{K}$-mean cluster 기법을 이용하여 군집 개수를 다양하게 변화시켜 가 면서 사용자의 인체크기를 적합하게 집단화하는 최적의 군 집 개수를 결정한다. 최적 군집 개수는 Figure 4에 예시적으 로 나타낸 것과 같이 군집의 중심과 군집에 소속된 사용자 간의 평균 거리가 수렴하는 수준(예: 20개 군집)에서 결정된 다(Lattin et al., 2003)
$\mathrm{EBZ}$ 기법은 군집 분석을 통해 설정된 최적 군집에서 1 명 씩의 사용자를 대표인체모델로 선정한다. 본 연구는 군집에 소속된 사용자 중에서 군집의 중심에 가장 가까운 사용자를 해당 군집의 대표자로 선정하였다. 예를 들면, 군집에 소속 된 사용자가 군집의 중심으로부터 $3 \mathrm{~cm}, 5 \mathrm{~cm}$, and $10 \mathrm{~cm}$ 떨어져 있으면, 군집 대표자는 군집의 중심에 가장 가까운 $3 \mathrm{~cm}$ 차이를 보인 사용자로 선정된다.

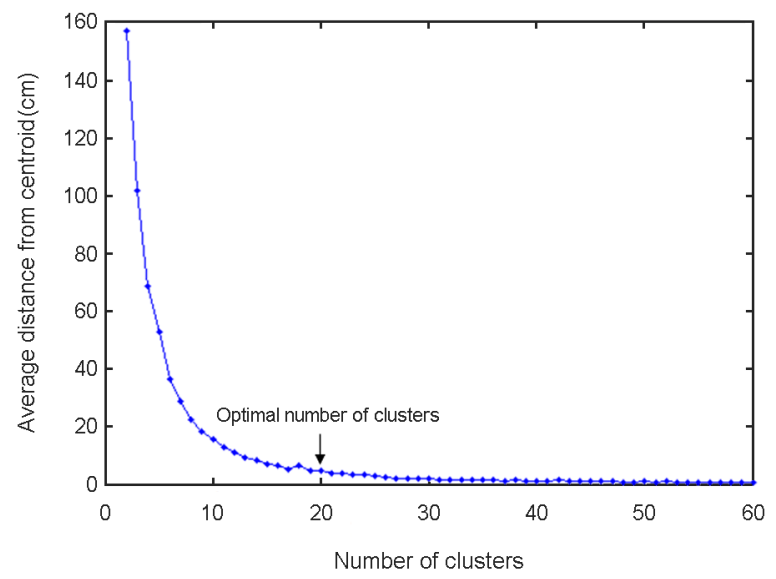

Figure 4. Illustration of an optimal number of clusters

\section{Performance Evaluation}

\subsection{Evaluation methods}

\subsubsection{Anthropometric data and variables}

본 연구는 $\mathrm{EBZ}$ 기법의 성능을 평가하기 위해 원시자료 확보가 용이한 US Army 인체측정자료 (Gordon et al., 1988)를 사용하였다. US Army 자료는 1988년에 미군 3,982 명 (남: 1,774 명, 여: 2,208명)을 대상으로 138 개 인체변수의 치수를 체계적으로 측정한 자료로서, 측정자 료가 방대하고 측정 신뢰성이 높다(Jung et al., 2009a). 그리고, US Army 자료는 인터넷에 공개되어 있어(http:// mreed.umtri.umich.edu/mreed/downloads.html) 인체측정 원시자료를 쉽게 확보할 수 있는 장점이 있다. 본 연구는 $\mathrm{EBZ}$ 기법을 적용한 대표인체모델 생성에 US Army 자료로 부터 계산된 기술통계자료를 이용하였으며, 생성된 대표인체 모델의 성능 평가에 US Army 원시자료를 활용하였다.

$\mathrm{EBZ}$ 기법의 성능 평가를 위한 인체변수는 현용 DHS 시 스템의 적용성을 고려하여 2개의 인체변수(키와 몸무게)가 사용되었다. 대표적 $\mathrm{DHS}$ 시스템인 $\mathrm{JACKO}^{\circledR}$ 은 키와 몸무게 
를 입력 받아 가상환경상 3 차원 인체모델을 생성한다(UGS, 2006). 본 연구는 $\mathrm{EBZ}$ 기법을 이용하여 생성된 대표인체모 델을 $\mathrm{JACK}^{\circledR}$ 에 연동시켜 사용할 수 있도록 키와 몸무게를 인체변수로 선정하였다.

\subsubsection{Performance measures}

본 연구는 3 가지 척도를 적용하여 $\mathrm{EBZ}$ 기법의 성능을 정 량적으로 평가하였다. 첫째, 수용률은 생성된 대표인체모델 의 인체크기가 설계 대상 사용자의 인체크기를 통계적으로 대표하는 정도를 나타낸다. 수용률은 Figure 5에 예시적으로 나타낸 것과 같이 대표인체모델이 포함하는 영역 내부에 위 치한 사용자의 비율로 계산되었다. 본 연구의 수용 영역은 대표인체모델을 모두 포함하는 최소 면적의 타원을 찾아주 는 최적화 모델(식 2; Moshtagh, 2006)을 이용하여 정의되 었다. 둘째, 이상치 비율은 대표인체모델의 인체크기가 설계 대상 사용자의 인체크기 범위를 벗어나는 비율로 계산된다 (Jung et al., 2009a). 마지막으로, 인체모델 개수는 대표인 체모델의 설계 적용 용이성을 나타내는 척도로서, 인체모델 의 개수가 적을수록 설계 및 평가에 적용이 용이함을 나타 낸다.

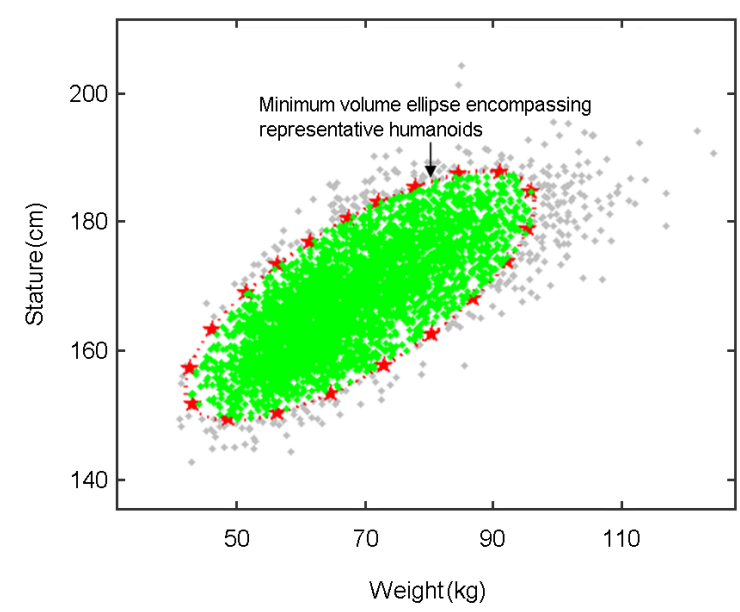

Figure 5. Illustration of a minimum volume ellipse encompassing representative humanoids(small dot: target users, dotted line: accommodation boundary line, star: representative humanoids)

$\min \log (\operatorname{det}(A))$

s.t. $\quad(p-c)^{\prime} A(p-c) \leq 1$

where: $A=$ n-by-n matrix of ellipse equation in the center form $p=\mathrm{d}$-by- $n$ matrix of body sizes of ebzRH $c=$ n-by-1 matrix of the center of the ellipse

\subsubsection{Evaluation procedure and program}

본 연구는 3 단계 절차를 통해 $\mathrm{EBZ}$ 기법의 성능을 체계적 으로 평가하였다. 첫째 단계는 US Army 자료의 키와 몸무 게에 대한 기술통계자료를 계산하였다. 둘째 단계는 계산된 기술통계자료를 $\mathrm{EBZ}$ 기법에 적용하여 대표인체모델을 100 회 반복 생성하였다. 마지막 단계는 생성된 100개의 대표인 체모델 집단별 성능을 US Army 원시자료를 이용하여 평가 하였다.

본 연구는 효율적인 평가 진행을 위해 Figure 6에 나타낸 것과 같은 평가용 소프트웨어를 Matlab으로 개발하여 사용 하였다. 개발된 소프트웨어는 US Army 인체측정자료에서 기술통계자료를 계산한 후 본 연구의 $\mathrm{EBZ}$ 기법을 적용하 여 대표인체모델을 생성해준다. 또한, 개발된 소프트웨어는 US Army 인체측정자료에 기반하여 생성된 대표인체모델의 수용률과 이상치 비율을 자동으로 계산해준다.

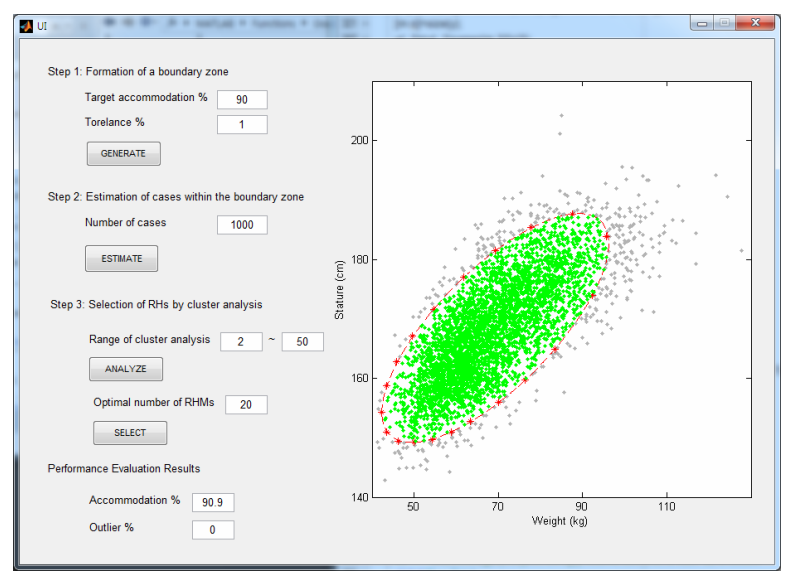

Figure 6. Generation and evaluation system of estimated boundary zone representative humanoids(small dot: target users, dotted line: accommodation boundary line, star: representative humanoids)

\subsection{Evaluation Results}

EBZ 기법의 수용률은 평균 90.9\%(범위: 90.8 91.1\%) 로 목표 수용률인 $90 \%$ 를 충족하는 것으로 분석되었다. $\mathrm{EBZ}$ 기법의 수용률은 Table 1 에 나타낸 것과 같이 BZ 기 법(91.0\%)과 유사한 것으로 나타났다. 또한, 반복에 따른 $\mathrm{EBZ}$ 기법의 수용률 표준편차는 $0.1 \%$ 로 나타나 BZ 기법 (표준편차: $0.6 \%$ ) 과 유사한 것으로 파악되었다.

$\mathrm{EBZ}$ 기법의 이상치 비율은 $\mathrm{BZ}$ 기법과 동일하게 $0 \%$ 인 것으로 분석되었다(Table 1 참조). 따라서 $\mathrm{EBZ}$ 기법은 설 
Table 1. Performance comparison between two generation methods of representative humanoids $(\mathrm{RH})$

\begin{tabular}{l|c|r|r}
\hline \multicolumn{2}{c}{ Measure } & \multicolumn{2}{c}{ Method } \\
\cline { 3 - 4 } \multicolumn{2}{c}{} & EBZ & BZ $^{*}$ \\
\hline $\begin{array}{l}\text { Accommodation } \\
\text { percent(\%) }\end{array}$ & Mean & 90.9 & 91.0 \\
\cline { 2 - 4 } & SD & 0.1 & 0.6 \\
\hline $\begin{array}{l}\text { Outlier proportion(\%) } \\
\text { Number of RH }\end{array}$ & Mean & 0.0 & 0.0 \\
\cline { 2 - 4 } & SD & 1.5 & 16.0 \\
\hline
\end{tabular}

*Source of data: Jung et al.(2009a)

계 대상 사용자의 인체크기 범위에 포함되는 대표인체모델을 생성한다. 이러한 EBZ 기법의 속성은 목표 수용률(90\%)과 허용률 $(1 \%)$ 을 이용해 형성된 경계 영역의 내부에 포함된 사용자 중에서 대표인체모델을 선정하기 때문이다.

마지막으로, EBZ 기법의 대표인체모델 생성 개수는 평균 20 명 (SD: 1.5 명)으로 나타나 BZ 기법의 16 명과 유사한 것 으로 분석되었다(Table 1 참조). 또한, $\mathrm{EBZ}$ 기법으로 생성 된 대표인체모델의 표준편차는 1.5 명으로 $\mathrm{BZ}$ 기법의 표준 편차(2.7명)와 유사한 것으로 파악되었다.

\section{Discussion}

본 연구는 기술통계자료를 이용하여 경계 영역 대표인체 모델을 추정하는 $\mathrm{EBZ}$ 기법을 개발하였다. BZ 기법(Jung et al., 2009a)은 목표한 비율을 통계적으로 수용하는 대표 인체모델을 생성할 수 있으나, 방대한 인체측정 원시자료를 필요로 하기 때문에 적용이 제한적이다. 본 연구는 $\mathrm{BZ}$ 기법 의 이론적 장점을 유지하면서 적용상의 한계점을 보완할 수 있는 $\mathrm{EBZ}$ 기법을 개발하였다. $\mathrm{EBZ}$ 기법은 다양한 인체크기 를 가지는 사용자를 수용하는 것이 중요한 자동차 운전석, 비행기 조종석, 그리고 작업 공간의 가상환경상 설계 및 평 가에 유용하게 활용될 수 있을 것으로 기대된다.

$\mathrm{EBZ}$ 기법은 인체크기의 정규분포 가정에 근간하여 목표 비율을 수용하는 경계 영역을 통계적으로 설정한다. 인체측 정 관련 연구들(Roebuck et al.(1975; Fernandez and Uppugonduri, 1992; Schoor and Konz, 1996)은 일반적 으로 인체크기의 분포를 정규분포로 가정하고 있다. 그러나 Vasu and Mital(2000)은 12개의 인체변수에 대한 정규성 검정을 통해 4 개(약 $33 \%$ ) 인체변수가 정규분포를 따르지 않는다고 보고하였다. 따라서 EBZ 기법의 이론적 타당성을 검증하기 위해 정규분포 가정의 위배에 따른 영향을 평가하
는 후속 연구가 필요하다.

본 연구의 EBZ 기법은 다수의 대표인체모델을 생성하는 것으로 나타났다. $\mathrm{EBZ}$ 기법은 경계 영역의 내부에 포함된 다양한 크기의 대표인체모델을 생성한다. 그로 인해, $\mathrm{EBZ}$ 기 법은 평균 20명의 대표인체모델을 생성하는 것으로 나타났 다. 대표인체모델의 개수가 많을 경우 사용자의 인체크기 다 양성을 보다 적합하게 대표할 수 있으나 $\mathrm{DHS}$ 를 이용한 제 품 평가에 투입되는 시간과 노력을 증가시키는 한계점이 있 다(Blome et al., 2006).

$\mathrm{EBZ}$ 기법의 활용성을 제고하기 위해서는 대표인체모델 개수가 많아도 효율적으로 가상환경상 설계 및 평가가 가능 한 DHS 시스템 개발이 필요하다. 대표적 DHS 시스템인 $\mathrm{Jack}^{\circledR}$ 은 수작업으로 대표인체모델 각각에 대한 평가를 수행 한다(Blome et al., 2006). 그로 인해, 대표인체모델 개수가 증가하면 선형적으로 설계 및 평가에 소요되는 시간이 증가 하게 된다. 따라서 대표인체모델 증가에 따른 분석 시간을 줄이기 위해서는 대표인체모델 1 명에 대한 분석 과정을 기 록하여 다른 대표인체모델에도 동일하게 적용시킬 수 있는 반자동 분석 기능을 개발하는 것이 요구된다. 이러한 반자동 분석 기능은 대표인체모델 개수가 증가하더라도 설계 및 평 가에 소요되는 시간이 선형적으로 늘어나는 한계점을 효과 적으로 보완할 수 있을 것이다.

\section{Acknowledgements}

This work was supported by the National Research Foundation of Korea Grant funded by the Korean Government (NRF2012R1A1A1011373).

\section{References}

Bittner, A.C., Glenn, F.A., Harris, R.M., Iavecchia, H.P. and Wherry, R.J. Cadre: A family of manikins for workstation design. In S.S. Asfour (Ed.) Trends in Ergonomics/Human Factors IV (pp. 733-740). North Holland: Elsevier Science Publishers, 1987.

Bittner, A.C. A-CADRE: Advanced family of manikins for workstation design. Proceedings of the IEA 2000/HFES 2000 Congress, 6, 774 $-777,2000$.

Blome, M., Hanson, L., Lämkull, D., Nielsen, E. and Stam, E., Identifying time-consuming human modeling tool activities. SAE Technical Paper Series (2006-01-2312), 2006.

Chaffin, D.B., Digital Human Modeling for Vehicle and Workplace Design. 
Pennsylvania, USA: SAE, 2001.

Fernandez, J.E. and Uppungouri, K.G., Anthropometry of south Indian industrial workmen. Ergonomics, 35, 1393-1398, 1992.

Gordon, C.C., Bradtmiller, B., Churchill, T., Clauser, C., McConville, J., Tebbetts, I. and Walker, R., 1988 anthropometric survey of U.S. Army personnel [Data file]. Retrieved from http://www.ntis.gov, 1988.

HFES 300 Committee. Guidelines for Using Anthropometric Data in Product Design. Santa Monica, CA: Human Factors and Ergonomics Society, 2004.

Jimmerson, D.G., 2001. Digital human modeling for improved product and process feasibility studies. In: Chaffin, D.B. (Ed.), Digital Human Modeling for Vehicle and Workplace Design (pp. 127-135). SAE International, Warrendale, PA, 2001.

Johnson, R.A. and Wichern, D.W., Applied Multivariate Statistical Analysis. New Jersey: Prentice Hall, 1988.

Jung, K., Kwon, O. and You, H., Development of the boundary zone method for generation of representative human models. Proceedings of the Human Factors and Ergonomics Society $53^{\text {rd }}$ Annual Meeting. Santa Monica, CA: The Human Factors and Ergonomics Society, 2009a.

Jung, K., Kwon, O. and You, H., Development of a digital human model generation method for ergonomic design in virtual environment. International Journal of Industrial Ergonomics, 39, 744-748, 2009b.

Kim, J. and Whang, M., Development of a set of Korean manikins. Applied Ergonomics, 28, 407-410, 1997.

Lattin, J.M., Carroll, J.D. and Green, P.E., Analyzing Multivariate Data. Pacific Grove, CA: Thomson Brooks/Cole, 2003.

Meindl, R.S., Hudson, J.A. and Zehner, G.F., A Multivariate Anthropometric Method for Crew Station Design (AL-TR-1993-0054). WrightPatterson Air Force Base, OH: Armstrong Laboratory, Air Force Material Command, 1993.

Meunier, P., Effects of a data reduction technique on anthropometric accommodation. Proceedings of the $42^{\text {nd }}$ Human Factors and Ergonomics Society, 727-731, 1998.

Moshtagh, N. (2006). Minimum volume enclosing ellipsoid. Retrieved from http://www.mathworks.co.kr/matlabcentral/fileexchange/9542minimum-volume-enclosing-ellipsoid.

Nelson, C., Anthropometric analyses of crew interfaces and component accessibility for the international space station. In: Chaffin, D.B. (Ed.), Digital Human Modeling for Vehicle and Workplace Design (pp. 17-36). SAE International,Warrendale, PA, 2001.

Porter, J.M., Case, K., Marshall, R., Gyi, D. and Oliver, R.S., Beyond Jack and Jill: designing for individuals using HADRIAN. International Journal of Industrial Ergonomics, 33, 249-264, 2004.
Roebuck, J.A., Kroemer, K.H.E. and Thomson, W.G., Engineering Anthropometry Methods. New York: Wiley-Interscience, 1975.

Ryu, T., Jung, I., You, H. and Kim, K., Development and application of a generation method of human models for ergonomic product design in virtual environment. Proceedings of the Human Factors and Ergonomics Society, 951-955, 2004.

Schoor, H.V. and Konz, S., Males/females: An anthropometric comparison for modeling missing data. International Journal of Industrial Ergonomics, 437-440, 1996.

Thompson, D.D., The determination of the human factors/occupant packaging requirements for adjustable pedal systems. In: Chaffin, D.B. (Ed.), Digital Human Modeling for Vehicle and Workplace Design (pp. 101-111). SAE International, Warrendale, PA, 2001.

UGS., Jack User Manual (Ver. 5.1). Unigraphics Corp, 2006.

Vasu, M. and Mital, A., Evaluation of the validity of anthropometric design assumptions. International Journal of Industrial Ergonomics, 26, 19 $-37,2000$.

\section{Author listings}

Kihyo Jung: kjung@ulsan.ac.kr

Highest degree: Ph.D., Department of Industrial and Management Engineering, POSTECH

Position title: Assistant Professor, School of Industrial Engineering, University of Ulsan

Areas of interest: Ergonomic product design, Digital human simulation, Usability testing, Work-related musculoskeletal disorders (WMSDs) Prevention

Date Received : 2013-02-12

Date Revised :2013-05-01

Date Accepted : 2013-05-15 